\title{
Rural children remain more at risk of acute malnutrition following exit from community based management of acute malnutrition program in South Gondar Zone, Amhara Region Ethiopia: a comparative cross-sectional study
}

\author{
Dereje B Abitew ${ }^{\text {Corresp., }}{ }^{,}$, Alemayehu W Yalew ${ }^{1}$, Afework M Bezabeh ${ }^{2}$, Alessandra N Bazzano ${ }^{3}$ \\ ${ }^{1}$ School of Public Health, Addis Ababa University, Addis Ababa, Ethiopia \\ 2 School of Public Health, Mekelle University, Mekele, Ethiopia \\ 3 Department of Global Community Health and Behavioral Sciences, School of Public Health and Tropical Medicine, Tulane University, New Orleans, LA, \\ United States \\ Corresponding Author: Dereje B Abitew \\ Email address: derejefrae2014@gmail.com
}

Background. Community based management of acute malnutrition has been reported effective in terms of recovery rate, but recovered children may be at increased risk of developing acute malnutrition after returning to the same household environment. Objective: Compare the magnitude and factors associated with acute malnutrition among recovered and never treated children in South Gondar Zone, Amhara Region, Ethiopia. Method. A comparative cross-sectional study was conducted in three districts of South Gondar Zone by tracing 720 recovered and an equal number of age matched children who were never treated for acute malnutrition. Parents were asked to bring children to health post for survey data collection, anthropometric measurements, and edema assessment. Data were collected using a survey questionnaire, entered in to EpiData and analyzed using SPSS v20. Anthropometric indices were generated according to the WHO's 2006 Child Growth Standards using WHO Anthro software version 3.2.2. Bivariate and multivariable logistic regression was utilized. Values with $P<0.05$ were considered statistically significant and Odds Ratio with $95 \% \mathrm{Cl}$ was used to measure strength of association. Result. Of 1440 parents invited, 1414 were participated (98.2\% response rate). Mean age in months of children ( \pm SD) was $23.7( \pm 10.4)$ for recovered and 23.3 ( \pm 10.8$)$ for comparison group. About $49 \%$ of recovered and $46 \%$ of comparison children were females. A significant difference was observed on magnitude of acute malnutrition between recovered $(34.2 \%(95 \% \mathrm{Cl} ; 30.9 \%-38.0 \%)$ and comparison groups $(26.7 \%$ (95\% Cl; 23.5-30.2\%), $\mathrm{P}=0.002$. Factors associated with acute malnutrition among recovered were district of Ebnat $(\mathrm{AOR}=3.7 ; 95 \% \mathrm{Cl}: 1.9$-7.2), Tach-Gayint $(\mathrm{AOR}=2.4 ; 95 \% \mathrm{Cl}$ : 1.2-4.7); male child $(A O R=1.4 ; 95 \% \mathrm{Cl}: 1.0-2.0)$; prelactal feeding $(\mathrm{AOR}=2.6 ; 95 \% \mathrm{Cl}: 1.3-5.1)$; not feeding colostrum $(\mathrm{AOR}=1.5 ; 95 \% \mathrm{Cl}: 1.1-2.3$ ); not consuming additional food during pregnancy/lactation $(\mathrm{AOR}=1.6 ; 95 \%$ $\mathrm{Cl}$ : 1.1-2.3); not given Vitamin A supplement ( $\mathrm{AOR}=2.1 ; 95 \% \mathrm{Cl}: 1.4-3.2)$; and safe child feces disposal practice $(A O R=1.7 ; 95 \% \mathrm{Cl}: 1.2-2.5)$ while district of Tach-Gayint ( $A O R=2.5 ; 95 \% \mathrm{Cl}$ : 1.3-4.8); male child $(A O R=1.5 ; 95 \% \mathrm{Cl}: 1.1-2.1)$, not feeding colostrum (AOR=1.7; 95\% Cl:1.2-2.5), poor hand washing practice $(A O R=1.6 ; 95 \% \mathrm{Cl}: 1.1-2.2)$; food insecure household $(A O R=1.6 ; 95 \% \mathrm{Cl}: 1.1-2.4)$, birth interval $<24$ months $(A O R=1.9 ; 95 \% \mathrm{Cl}, 1.2-3.2)$, and poor access to health facility $(\mathrm{AOR}=1.7 ; 95 \% \mathrm{Cl}: 1.2-2.4)$ were factors associated with acute malnutrition among comparison group. Conclusion. Recovered children were more at risk of acute malnutrition than the comparison group. Nutrition programs should invest in improving nutrition counseling and education; as well as the hygienic practices to protect children against post-discharge relapse of acute malnutrition.

Peer) reviewing PDF | (2019:08:40536:2:0:NEW 8 Dec 2019) 
1 Rural children remain more at risk of acute malnutrition following exit from community based

2 management of acute malnutrition program in South Gondar Zone Amhara Region Ethiopia: a

3 comparative cross-sectional study

4 Dereje Birhanu Abitew ${ }^{1 *}$, Alemayehu Worku Yalew ${ }^{1}$, Afework Mulugeta Bezabih ${ }^{2}$, Alessandra

5 N. Bazzano ${ }^{3}$

$6{ }^{1}$ School of Public Health, Addis Ababa University, Addis Ababa, Ethiopia,

$7 \quad{ }^{2}$ School of Public Health, Mekelle University, Mekele, Ethiopia

$8{ }^{3}$ Department of Global Community Health and Behavioral Sciences, School of Public Health and

9 Tropical Medicine, Tulane University, New Orleans, LA, USA

10 The corresponding author:

11 Dereje Abitew*

12 Addis Ababa

13 Ethiopia

14 derejefrae2014@gmail.com

\section{Abstract}

16 Background. Community based management of acute malnutrition has been reported effective in terms of recovery rate, but recovered children may be at increased risk of developing acute malnutrition after returning to the same household environment.

19 Objective: Compare the magnitude and factors associated with acute malnutrition among recovered and never treated children in South Gondar Zone, Amhara Region, Ethiopia.

Method. A comparative cross-sectional study was conducted in three districts of South Gondar Zone by tracing 720 recovered and an equal number of age matched children who were never treated for acute malnutrition. Parents were asked to bring children to health post for survey data collection, anthropometric measurements, and edema assessment. Data were collected using a survey questionnaire, entered in to EpiData and analyzed using SPSS v20. Anthropometric indices were generated according to the WHO's 2006 Child Growth Standards using WHO Anthro software version 3.2.2. Bivariate and multivariable logistic regression was utilized. Values with $\mathrm{P}<0.05$ were considered statistically significant and Odds Ratio with 95\% CI was used to measure strength of association.

30 Result. Of 1440 parents invited, 1414 were participated (98.2\% response rate). Mean age in months of children $( \pm \mathrm{SD})$ was $23.7( \pm 10.4)$ for recovered and $23.3( \pm 10.8)$ for comparison 
32 group. About $49 \%$ of recovered and $46 \%$ of comparison children were females . A significant 33 difference was observed on magnitude of acute malnutrition between recovered (34.2\% (95\% CI;

$3430.9 \%-38.0 \%)$ and comparison groups $(26.7 \%(95 \% \mathrm{CI} ; 23.5-30.2 \%), \mathrm{P}=0.002$. Factors 35 associated with acute malnutrition among recovered were district of Ebnat (AOR $=3.7 ; 95 \% \mathrm{CI}$ : 1.9 -7.2), Tach-Gayint (AOR=2.4; 95\% CI: 1.2-4.7); male child ( $\mathrm{AOR}=1.4$; 95\% CI: 1.0-2.0); prelactal feeding $(\mathrm{AOR}=2.6$; 95\% CI:1.3 -5.1); not feeding colostrum $(\mathrm{AOR}=1.5 ; 95 \% \mathrm{CI}: 1.1$ 2.3 ); not consuming additional food during pregnancy/lactation ( $\mathrm{AOR}=1.6$; $95 \% \mathrm{CI}: 1.1-2.3$ ); not given Vitamin A supplement ( $\mathrm{AOR}=2.1 ; 95 \% \mathrm{CI}: 1.4-3.2)$; and safe child feces disposal practice ( $\mathrm{AOR}=1.7 ; 95 \% \mathrm{CI}: 1.2-2.5)$ while district of Tach-Gayint ( $\mathrm{AOR}=2.5 ; 95 \%$ CI: 1.3-4.8); male child ( $\mathrm{AOR}=1.5 ; 95 \% \mathrm{CI}: 1.1-2.1$ ), not feeding colostrum ( $\mathrm{AOR}=1.7 ; 95 \% \mathrm{CI}: 1.2-2.5)$, poor hand washing practice ( $\mathrm{AOR}=1.6 ; 95 \% \mathrm{CI}$ : 1.1-2.2); food insecure household ( $\mathrm{AOR}=1.6$; 95\% CI: 1.1-2.4), birth interval $<24$ months (AOR=1.9; 95\% CI,1.2-3.2), and poor access to health facility $(\mathrm{AOR}=1.7 ; 95 \%$ CI:1.2-2.4) were factors associated with acute malnutrition among comparison group.

Conclusion. Recovered children were more at risk of acute malnutrition than the comparison group. Nutrition programs should invest in improving nutrition counseling and education; as well as the hygienic practices to protect children against post-discharge relapse of acute malnutrition.. Keyword. Acute malnutrition, child relapse, community based management program, wasting, Ethiopia

\section{Introduction}

Under-nutrition is the end result of nutrient deprivation either due to lack of adequate intake or repeated infection (1) and is categorized as either chronic or acute malnutrition based on duration of deprivation (2). Acute malnutrition is further classified as either moderate acute malnutrition (MAM) or severe acute malnutrition (SAM) based on the degree of malnutrition and the presence of edema (2). According to a 2018 report, globally over 49 million children under 5 were wasted and nearly 17 million were severely wasted (3) while the magnitude nationally in Ethiopia and in Amhara region was found 7.25 and $7.6 \%$ (4) and also it was $11.8 \%$ in the study area (5). Health consequences for children suffering from wasting include weakened immunity and increased risk of death, particularly when wasting is severe (3). Mortality rate due to SAM is between 5-20 times higher compared with mortality of well-nourished children $(2,6)$. Globally about 1 million children die every year from SAM and $(7,8)$ and in Ethiopia about $57 \%$ of all 
63 under-five deaths are related to malnutrition, of which three-quarters result from complications 64 associated with mild to moderate malnutrition (9). In addition about $70 \%$ of all childhood

65 66 mortality in developing countries is due to five major conditions, for which malnutrition (even in mild form) increases the likelihood of mortality up to $56 \%$ (7). In order to address nutrition related public health problems, ten high-impact, nutrition-specific interventions have been identified that, if taken as a package up to 90 percent coverage, could reduce wasting by 60 percent, including the management of SAM and MAM as 2 of the 10 interventions (10). Children with uncomplicated SAM (WHZ below -3 SD cut-off and/or with mid upper arm circumference (MUAC) cut-off of $115 \mathrm{~mm}$ and/or with bilateral edema) and MAM children (WHZ between -2 and -3 or MUAC between 115 and 125 millimeters) may be treated in the community setting without requiring admission to a health facility. This process is referred to as Community based Management of Acute Malnutrition (CMAM) $(11,12)$ and consists of providing a child with special therapeutic foods, most commonly Ready-to-Use- Therapeutic Food (RUTF) or F75 and F100 milk-based diets, based on severity and a short course of basic oral medication to treat infections. (2). According to the WHO recommendation, children with SAM are discharged as recovered from treatment when their weight-for-height/length is $\geq-2 \mathrm{Z}$ score and they have had no edema for at least 2 weeks, or mid-upper-arm circumference is $\geq 125 \mathrm{~mm}$ and they have had no edema for at least 2 weeks. Children admitted with only bilateral pitting edema should be discharged from treatment based on whichever anthropometric indicator, mid-upper arm circumference or weight-for-height is routinely used in the local program (13). In the current Ethiopian CMAM program, the admission criteria for children age 6 months to 18 years are: $\mathrm{W} / \mathrm{H}$ or $\mathrm{W} / \mathrm{L}<70 \%$, or MUAC $<110 \mathrm{~mm}$, or presence of bilateral pitting edema. The local national discharge criteria are: $\mathrm{W} / \mathrm{H} \geq 85 \%$ or $\mathrm{WFH} \geq-2 \mathrm{Z}$ score for two consecutive weeks (14days) and no oedema for 14 days (if admitted with edema) and the second option is target weight gain for two consecutive visits and no edema for 14 days (if admitted with edema) (14). Community based management of acute malnutrition programming was established to treat severely acutely malnourished (SAM) children in their communities without being admitted to a health facility $(11,15)$ has been reported effective in terms of the three key performance indicators: recovery, default and death rate (12) In some research findings, the recovery rate has been reported above the Sphere Handbook (12) minimum standards of $>75 \%(16,17)$ but post-discharge relapse rates of acute malnutrition 
94 (severe and moderate) have been reported as high as 78\% (69\% for MAM \& 9\% for SAM) in 95 Bangladesh (18) 27\% (10\% SAM \& 17\% MAM) in Malawi (19) and 72.1\% (34.6\% SAM \& $9637.5 \%$ MAM) in Southern Ethiopia (20).

97 Children discharged after recovery needs to be followed longitudinally to assess improvement 98 over time. The Ethiopian Federal Ministry of Health has recommended one to two months follow 99 up (14), but poor post-discharge follow up has been reported (21) and based on current findings

100 from the literature (22), it is plausible that acutely malnourished children who were discharged as 101 recovered may be predisposed to develop acute malnutrition (either MAM or SAM) again after 102 returning to the same household $(\mathrm{HH})$ environment.

103 Despite emerging data, a gap continues to exist in the evidence base on the differences in acute 104 malnutrition between children who have been treated using CMAM and those who have not. The 105 objective of the current study was to compare the magnitude and factors associated with acute 106 malnutrition between children following exit from CMAM compared with those who were never 107 treated for acute malnutrition in South Gondar Zone of Amhara Region, Ethiopia

108 Method and materials

109 Study area and setting

110 The study was conducted in South Gondar Zone of Amhara region, Ethiopia. South Gondar Zone 111 is one of 11 administrative zones of Amhara region, Ethiopia. Debretabor is the capital city of

112 the zone situated about $100 \mathrm{Km}$ East of Bahir Dar (the capital city of Amhara Region) and 667

$113 \mathrm{Km}$ North of Addis Ababa (the capital city of Ethiopia). The Zone has 17 districts, five of which 114 are town administrations. According to the Central Statistical agency of Ethiopia, the 2017/18

115 population of the Zone was 2,484,929 of which 183, 525 were children 0-4yrs old (23). In South

116 Gondar, there are one Zonal and three district hospitals, 90 Health Centers, 378 Health Posts, and 117 more than 10 private clinics. According to the Zonal health department annual report, $68 \%$ of 118 children under two years of age had received growth monitoring program, and a total of 6,468

119 SAM children were managed in the health facilities with an overall $96.8 \%$ recovery rate $(24)$.

120 Study design

121 A comparative cross-sectional study was conducted from 10 Nov 2017 to 30 Jan 2018 in South

122 Gondar Zone of Amhara Region, Ethiopia. 


\section{Study Population}

124 The study population for the recovered group was children $6-59$ months old following exit

125 from CMAM in the randomly selected districts of South Gondar Zone and for the comparison

126 group, the study population were 6-59 months old children who were never treated for SAM in

127 the randomly selected districts of South Gondar Zone.

128 Exclusion criteria:

129 Children were excluded if their name was different from the name present in the CMAM

130 registration logbook and/or suffering from any systemic disease.

\section{Sample size determination and procedure}

132 Since the main objective of this study was to assess any difference in the magnitude of acute

133 malnutrition (wasting) among children following exit from CMAM as compared with children

134 who had never been treated for SAM in the CMAM program, two proportion formula was used.

135 The sample size was calculated using Epi Info version 7.1.3.3 taking percent of acute

136 malnutrition among children following exit from CMAM 78\% as described in the literature (18)

137 and assumption of a 10\% difference in percentage of acute malnutrition in the general population

138 (children age 6-59 months) who were never treated for SAM with 95\% CL, 80\% power, and a

139 ratio of $1: 1$, the sample size calculated was 656 and when multiplied by a design effect of 2 and

140 including 10\% non-response rate, the sample size was 1440 (720 recovered and 720 from the

141 comparison group).

142 Regarding the sampling procedure, a multistage sampling technique was used. Among the 17

143 districts of the Zone, five were town administrations and the remaining 12 were rural

144 administrative districts. From these rural districts, three districts were selected randomly using

145 lottery method and from these three districts, 10 health centers were selected randomly. In the

146 current Ethiopian health system, a health Centre has five cluster health posts in its catchment

147 area and we considered three to five health posts per health Centre based on the caseload report

148 from the CMAM program. Therefore, 26-43 discharged recovered children were expected per

149 health post. Since this was part of a larger study, a sampling framework developed using the one-

150 year therapeutic multi-chart logbook (from Nov 2016 to Oct 2017) to trace 1290 discharged

151 recovered children was used to select these 720 discharged recovered children taking every

152 second child as a study participant. 
153 An age-matched ( \pm 6 months) child who was never treated for SAM but was visiting the health 154 post either for immunization, growth monitoring program, and/or clinical services was selected

155 for the comparison group. Finally, parents of recovered children were contacted by Health

156 Extension Workers (HEWs) and asked to bring children to the health post for survey data

157 collection, anthropometric measurements, and edema assessment.

158 Study variables

159 Dependent variable: Acute malnutrition among children age 6-59 months in South Gondar Zone, 160 Amhara region, Ethiopia.

161 Independent variables: socio-economic and demographic, household hygiene/sanitation, child 162 caring/feeding knowledge/practice, health facility access, and household food security related 163 variables.

\section{Data collection tools and measurements}

165 The data collection tools consisted of a checklist and a questionnaire. The checklist was prepared

166 using the Stabilization Centre/outpatient therapeutic program (SC/OTP) multi-chart and

167 registration logbook utilized nationally $(2,14)$ to identify and trace discharged recovered

168 children. In addition, an interviewer-administered questionnaire was prepared following a review

169 of pertinent literature $(4,19)$ to address the socioeconomic, demographic, child feeding/caring

170 practices, and health and nutrition-related characteristics of respondents. The questionnaire was

171 prepared in English and then translated to the local language (Amharic) and back to English to

172 check the consistency. Mothers or primary caretakers were interviewed using the questionnaire

173 which took approximately 20-30 minutes. The data collection took place from 10 Nov 2017 to 30

174 Jan 2018.

175 To identify history of morbidity of children, mothers were asked about any occurrence of illness

176 during the past two weeks and were probed by the enumerator to confirm the nature of the illness

177 based on operational case definitions. Children's vaccination status was checked by observing an

178 immunization card and if not available, mothers were asked to recall child vaccination in as

179 much detail as possible: BCG vaccination was checked if any scar existed on a child's arm.

180 A total of 15 data collectors who had SAM management training and relevant previous

181 experience in data collection were recruited and trained for two days, with training content

182 mainly focusing on anthropometric measurement techniques and on how to administer the

183 questionnaire $(2,14)$. The data collectors were closely supervised by the three trained 
184 supervisors (public health, nurse or environmental health professionals) and also by the principal 185 investigator.

186 Anthropometric measurements

187 Child MUAC was measured halfway between the olecranon and acromion process using non

188 stretchable tape following the standard procedure and recorded to the nearest $0.1 \mathrm{~cm}(2)$.

189 Measurements were taken twice and a $0.1 \mathrm{~cm}$ variation between the two was accepted as normal.

190 However, repeated measurements were carried upon significantly larger variations according to

191 the stated protocol(25).

192 In addition, presence of edema was assessed by grasping both feet in the hands with the thumbs

193 on top of the feet and then pressing the thumbs gently for three seconds or a count of

$194101,102,103$ and then releasing the thumbs. It was registered as " 0 " if no pitting was detected on

195 the feet, recorded as "+" if an indent was detected on feet, "++" if on legs and feet, and "+++" if

196 it included the hands and face according to accepted standards $(2,14)$.

\section{Operational/definition of terms}

198 For the current study, acute malnutrition was defined based on child MUAC $<12.5 \mathrm{~cm}$ and/or

199 presence of edema, not acutely malnourished otherwise (2)

200 Good hand washing practice: A respondent was categorized as having good hand washing

201 practice if they reported washing hands at three of four critical times/points (before eating,

202 before preparing food, after defecation, and after cleansing child feces).

203 Safe child feces disposal: Respondent's child feces disposal practice was considered safe if the 204 child uses a latrine or if the caregiver placed child feces in a latrine.

205 Improved water source: A household was considered to have improved drinking water if the 206 source was either from a pipe, protected spring, protected well and/or boiled water.

207 Currently on FP: A respondent was considered as currently using family planning if she 208 reported having used any family planning methods to avoid pregnancy or extend the interval 209 between births.

210 HH food insecurity access scale: Household food insecurity status was determined using the

211 nine item Household Food Insecurity Access Scale question and prior to assigning the food

212 insecurity (access) category, each frequency of occurrence question was coded as 0 for all cases

213 where the answer to the corresponding occurrence question was "no" and then the four food 
214 security categories were created sequentially as recommended by FANTA (26). Finally, the

215 HFIA scale category one (I) was considered as food secure and the remaining as food insecure.

216 Data management and analysis

217 The questionnaire was checked manually for completeness and was entered into EpiData version

218 3.3.2, and exported to SPSS version 20 for analysis. Anthropometric indices were generated

219 according to the WHO's 2006 Child Growth Standards (27) using the WHO Anthro software

220 version 3.2.2.

221 Since the study was comparative, a chi-square test was performed to detect any significant

222 difference in the prevalence of acute malnutrition between the two groups. Univariate analysis

223 was done to see if any association between the outcome and the independent variables and those

224 variables with $\mathrm{P}$ value $<0.2$ were entered into the final regression model. Variables with $\mathrm{P}$ value

$225<0.05$ were considered as predictors of acute malnutrition and strength of association was

226 determined using odds ratio with 95\% CIs. The final model was tested for goodness of fit using

227 Hosmer-Lemeshow test and the standard error (SE) values of variables in the final model were

228 checked for multicollinearity.

229 Data quality assurance

230 To assure data quality, data collectors and supervisors were trained for two consecutive days on

231 anthropometric measurements techniques based on the stated standard $(2,14)$ and were closely

232 supervised by supervisors and investigators. Pre-testing took place in a health post from a non-

233 study district of similar setting to the study sites. Anthropometric tools were checked daily by

234 setting the scale to zero and by measuring a known object (usually IV fluid) to check its

235 accuracy.

236 Ethical statement

237 The protocol was approved by the institutional review board (IRB) of the College of Health

238 Sciences of Addis Ababa University with an approval number of 068/16/SPH, and permission

239 letters were obtained from Regional, Zonal and District health offices of Amhara region,

240 Ethiopia. Informed verbal consent was obtained from all study respondents. Data collectors link

241 children to OTP if child MUAC was $<11.0 \mathrm{~cm}$, and $/$ or the child had edema. Nutrition counseling

242 was given to mothers/caretakers after the interview. 


\section{Result}

\section{Socioeconomic and demographic characteristics of respondents}

245 Overall there were 1440 respondents, of which, 1414 (707 recovered \& 707 from the 246 comparison groups) were interviewed (98.2\% response rate). All the respondents (i.e.

247 parents/mothers of children) from the two groups were female by gender, rural by residence, and 248 Amhara by ethnicity. The mean age $( \pm \mathrm{SD})$ in months of children was $23.7( \pm 10.4)$ for the 249 recovered and $23.3( \pm 10.8)$ for the comparison group. About $44 \%$ of recovered and $40 \%$ of 250 comparison children age ranged from 12-23 months. In addition, $49 \%$ of the recovered and $46 \%$

251 of the comparison were female children. The majority (93\%) of the respondent's religion in both 252 groups was Orthodox Christians, 94\% in the recovered and 90\% in the comparison group were 253 currently married. Nearly $60 \%$ of respondents in the two groups were unable to read and write.

254 In addition, $81 \%$ in the recovered and $77 \%$ in the comparison groups were farmers by 255 occupation. About equal (23\% of recovered \& $25 \%$ of comparison group) number of HHs was 256 food insecure. Moreover, about one in six (15\%) and one in four (27\%) of HHs were in the 257 lowest wealth quintile as indicated in (Table 1).

258 Child feeding/caring and housing conditions

259 About four fifths of children in the two groups (79\% of recovered and $81 \%$ of comparison group) 260 were reported breastfed and $73 \%$ of the mothers in the recovered and $71 \%$ in the comparison 261 group reported providing colostrum to the child. The water source for drinking was improved for $26256 \%$ of the recovered \& $58 \%$ of the comparison group HHs. About half $(50.9 \%$ in the recovered $263 \& 48.8 \%$ in the comparison group) of HHs reported discarding dry human waste into open fields.

264 The majority of children (96\% in the recovered and $85 \%$ in the comparison group) were 265 vaccinated for measles, and $76 \%$ of children in the recovered and $62 \%$ in the comparison group 266 received Vitamin A supplementation in the six months preceding the survey (Table 2).

267 Prevalence of acute malnutrition

268 Regarding child nutritional status, a significant difference was observed in the magnitude of 269 acute malnutrition (MUAC $<12.5 \mathrm{~cm}$ ) between the recovered (34.4\%, 95\% CI; $30.9-38.0)$ and the 270 comparison groups $(26.7 \%, 95 \% \mathrm{CI} ; 23.5-30.2) \mathrm{P}=0.002$. From these, $12.0 \%$ of the recovered 271 and $9.5 \%$ of the comparison group were severely acutely malnourished (MUAC $<11.5 \mathrm{~cm}$ ). In 272 addition, 66.9\% (95\% CI, 63.3-70.4) of children in the recovered and 58.3\% (95\% CI, 54.5-61.9)

273 of children in the comparison group were stunted (HAZ $<-2$ SD) see (Table3 and Figure 1) 
274 Factors associated with acute malnutrition

275 Regarding factors associated with acute malnutrition, about 28 variables for the recovered, and

27616 for the comparison who had $\mathrm{P}$ value $<0.2$ in the univariate analysis were entered in to the

277 final regression model. Eight variables from the recovered and seven from the comparison group

278 retained their statistical significance at $\mathrm{P}<0.05$. The odds of relapse in the recovered children

279 were more than three times $(\mathrm{AOR}=3.67 ; 95 \% \mathrm{CI}, 1.86-7.24)$ higher among children who lived at

280 Ebnat and two times (AOR=2.36; 95\% CI, 1.20-4.69) higher among children who lived at Tach-

281 Gayint district compared to those who lived in Lay-Gayint district. The odds of acute

282 malnutrition were about 1.5 times higher in male than female children ( $\mathrm{AOR}=1.43$; $95 \% \mathrm{CI}$,

283 1.01-2.01) and more than two times higher among children who were fed prelacteal food than

284 children who did not receive prelacteal feeding ( $\mathrm{AOR}=2.56$; 95\% $\mathrm{CI}, 1.28-5.12$ ).

285 Similarly, the odds of acute malnutrition in children from the comparison group were 2 times

$286(\mathrm{AOR}=2.49 ; 95 \% \mathrm{CI}, 1.29-4.81)$ higher among children who lived at Tach-Gayint compared to

287 those who lived at Lay-Gayint district. The odds of acute malnutrition were 1.5 times higher in

288 male than female children ( $\mathrm{AOR}=1.47 ; 95 \% \mathrm{CI}, 1.02-2.11)$ and about 1.6 times higher among

289 children in food insecure than food secure HHs (AOR=1.59; 95\% CI, 1.06-2.38). In addition, the

290 odds of acute malnutrition were two times higher among children with birth interval of $<24$

291 months than those with birth interval above $>=24$ months (AOR=1.92; 95\% CI, 1.16-3.19), and

2921.5 times higher among children whose mothers had practiced hand washing only after one or

293 two of the key points compared to those who wash hands more frequently (AOR $=1.55 ; 95 \% \mathrm{CI}$,

294 1.08-2.21) (table 4).

295 Discussion

296 In the current study, the prevalence of acute malnutrition was higher in the recovered than the

297 comparison group.

298 Some health and nutrition organizations have reported that recovery rates from malnutrition are

299 above the Sphere (12) minimum standard of $>75 \%(16,17,28)$ indicating good progress, but

300 recovered children may still be in a state of heightened risk for acute malnutrition. One possible

301 issue is lack of follow up intervention after discharge as recovered. The Ethiopian Federal

302 Ministry of Health has recommended linking to a supplementary feeding program for another 4

303 months following exit from therapeutic programs (14). But in the current study, none of the

304 children were linked to supplementary feeding programs, so were likely at high risk of relapse. 
305 Studies have reported that children who were treated for 12 weeks after fulfilling discharge 306 criteria had lower rates of SAM (29), similarly children who continued supplementary feeding

307 longer than the required time remained well-nourished over the subsequent year (19). An

308 additional potential factor could be due to the changes in the eating habits of children. Children

309 who were in the program receiving therapeutic food (ready to use therapeutic food (RUTF),

310 F100, and/or F75) may not be willing to return to receiving family food as frequently as before

311 admission to the program. In addition, these recovered children may go through repeated

312 episodes of treatment with RUTF (6) and such increased consumption could lead them to have a

313 modification of taste preference towards sweet or fatty foods (30) s. In addition, even though

314 the long-term impact of consumption of RUTF on the gut microbiome of SAM children is not

315 yet fully understood, there is some evidence that microbiota are easily manipulatable by dietary

316 changes (31) and this has been implicated as a causal factor of kwashiorkor (32) .

317 Regarding the factors identified to be associated with acute malnutrition, eight variables (district

318 of residence, sex of the child, prelacteal feeding, colostrum feeding, Vitamin A supplementation,

319 child feces disposal practice, respondent age in years., and consumption of additional food

320 during pregnancy/lactation) in the recovered group and seven variables (district of residence, sex

321 of the child, colostrum feeding, HH food security status, home to health post distance,

322 respondent hand washing practice, and birth interval) in the comparison groups were identified

323 as predictors of acute malnutrition. Three of these predictors (district of residence, sex of the

324 child, and colostrum feeding) were common in both groups.

325 In the current study, sex of the child was the factor associated with acute malnutrition. Male

326 children both in the recovered and in the comparator group were more acutely malnourished than

327 female children. This finding was in line with studies conducted in Malawi (19, 29), Sierra

328 Leone (33), Tanzania (34), Nigeria (35), Burkina Faso (36) and Ethiopia (37, 38). One possible

329 factor could be the typical activities of boys in to be away from home performing tasks leading

330 to missed meals. A recent study has also noted that boys health may be more influenced by

331 environmental stressor and diarrhea (39).

332 District of residence was a factor associated with acute malnutrition both in the recovered and 333 comparison groups. Other studies reporting that geographic variation was associated with acute

334 malnutrition in Bangladesh in relation to Eastern versus Southern district (40) and in Sylhet

335 versus Dhaka division (41) as well as in Zambia between Northern versus Western provinces 
336 (42). This could be linked to malaria transmission by geographic area (43), or The difference 337 could also be related to child food preparation practice (as one third of mothers from Lay338 Gaying versus one tenth from Ebnat). A study in Afar region, Ethiopia found that children's food 339 wasn't prepared separately from family food were more acutely malnourished (44)

340 Children both in the recovered and in the comparison groups who were not given colostrum were

341 found more acutely malnourished than those who were given colostrum. This is because 342 colostrum has all the essential nutrients and immunoglobulin that are important in disease 343 prevention. The finding was in line with studies from India (45) and Ethiopia (46). Practice of 344 colostrum feeding may be related to maternal education/counseling on child nutrition/health as 345 those who had good nutrition knowledge had better infant feeding practices $(47,48)$ and to have 346 more well-nourished children (49). The practice of EBF may also linked, as exclusively breast 347 fed children were less acutely malnourished than their counterparts $(50,51)$.

348 Children who were given prelacteal feeding were more acutely malnourished than those who 349 were not. The finding echoes other reports from India $(45,52)$, and Ethiopia (37). Prelacteal 350 feedings disrupts the feeding of colostrum, the practice of EBF, and increase likelihood of other 351 foods being introduced before 6 months, as has been reported in Ethiopia and Vietnam $(53,54)$.

352 This also results in enteric infections and environmental enteropathy due to consumption of 353 unsafe water or liquids (55).

354 The odds of relapse were higher among children not given high dose Vitamin A supplementation 355 in the 6 months preceding the survey. This may indicate low receipt of, or access to nutrition 356 services such as distribution of Vitamin A capsule and has also been noted in Hawassa, and Afar 357 region, Ethiopia $(44,56)$. The protective role of Vitamin $\mathrm{A}$ in promoting and regulating 358 activities in both the innate and adaptive immune system (57) enhancing immune function is the 359 rationale for recommended supplementation every 6 months (58).

360 International recommendations for pregnant and lactating women include at least one extra 361 serving of food per day to meet extra caloric needs (59). The finding was in agreement with a 362 study conducted in East Gojjam, Ethiopia describing more wasting among children whose 363 mothers' were not able to consume extra food during pregnancy (51). Undernourished 364 (BMI <18.5) mothers are more likely to have undernourished children $(40,52)$.

365 Safe child feces disposal is important in reducing diarrheal diseases and malnutrition, , but in the 366 current study, more acutely malnourished children were identified in HHs who reported 
367 practicing safe child feces disposal than in those who dispose in to open field/bush. A study in

368 Zambia noted that children who always used latrine were more wasted than those whose feces

369 was buried (60). Yet a study in India reported that those children who utilize latrine were less

370 acutely malnourished (52). Authors in Indonesia reported that those who disposed of child stool

371 safely had fewer episodes of diarrhea (61). Availability of latrine facilities and/or assuming safe

372 child feces disposal is therefore not a guarantee unless it is been practiced in an optimal

373 condition.

374 Unsurprisingly, the odds of acute malnutrition among children in food insecure HHs were higher

375 than in food secure HHs. The finding was in agreement with studies conducted in India (62),

376 Nigeria (63), and Ethiopia (56) Acute malnutrition in food insecure HHs can also be linked to

377 inadequate intake of diversified foods as studies have reported that consumption of low dietary

378 diversity food ( $\leq 3$ food groups) as being associated with acute malnutrition (64-66). Other

379 studies also pointed to the role of low socioeconomic status or monthly income in food insecurity

$380(49,66)$ which directly or indirectly reduces the HH purchasing power, and thus, reduces access

381 to food.

382 Odds of acute malnutrition were higher among children whose mothers had practiced hand

383 washing only after one or two of the key points compared to those who washed hands more

384 frequently. The World Health Organization (WHO) recommends washing hands at four key time

385 points : before eating, before preparing food, after defecation, and after disposal of child feces

386 (67)., Our finding are similar to other studies where caregivers who washed hands regularly

387 were less likely to have acutely malnourished children $(46,52,65)$. Hand washing reduces the

388 risk of contamination by excreta and thereby transmission of pathogens(68). One of the strength

389 of this study is that this is the first cross-sectional study employing a comparative (control) group

390 in Ethiopia to better understand if relapse rate differed following exit compared with non- treated

391 cohorts. Despite standardization of anthropometric instruments, intensive training, and close

392 supervisions, misclassification of children's nutritional status due to measurement error is

393 potentially a limitation. Children treated for SAM before could be considered as never treated

394 due to caretakers recall bias and also their children immunization status. Inclusion of children

395 attending for clinical services could overestimate the burden of wasting. In addition, $\mathrm{HH}$ food

396 insecurity status may be underestimated, as the data collection time was in the harvest season

397 (October 2017 to January 2018). 


\section{Conclusion and recommendation}

399 In the present study, recovered children were more at risk of acute malnutrition than the

400

401

402

403

404

405

406

407

408

409

410

411

412

413

414

415

416

417

418

419

420

421

422

423

424

425

426

427

428

429

430

431

432

433

434

435

436

comparison group. Three variables, (district of residence, sex of the child and not feeding

colostrum) were common predictors in the two groups. Understanding the factors associated with

acute malnutrition may help improve in prevention and management of future post discharge

relapse. Nutrition programs should invest in improving nutrition counseling and education

especially focusing on IYCF practices; as well as the hygienic practices to protect children

against post-discharge relapse of acute malnutrition.

\section{Acknowledgments}

Our appreciation goes to the Amhara Regional Health Bureau, South Gondar Zone Health

Department, and all three district health office heads \& nutrition focal persons. We also

especially thank study participants, supervisors and data collectors.

\section{References}

1. WHO. Management of severe Malnutrition: A manual for physicians And other senior health Workers. 1999.

2. WHO, UNICEF. WHO Child growth standards and the identification of severe acute malnutrition in infants and children, A Joint statement by the World Health Organization and the United Nations Children's Fund; World Health Organization Press: Geneva, Switzerland. 2009.

3. UNICEF, WHO, Bank W. Levels and trends in child malnutrition: Key findings of the 2018 Edition of the Joint Child Malnutrition Estimates. 2018.

4. EDHS. Ethiopian Public Health Institute (EPHI) [Ethiopia] and ICF. Ethiopia Mini Demographic and Health Survey : Key Indicators. Rockville, Maryland, USA: EPHI and ICF. 2019.

5. Motbainor A, Taye A. Wasting in under five children is significantly varied between rice producing and non-producing households of Libokemkem district, Amhara region, Ethiopia. BMC pediatrics. 2019;19(1):300.

6. WHO, WFP, Nutrition USSco, UNICEF. Community-based management of severe acute malnutrition; A Joint Statement by the World Health Organization, the World Food Programme, the United Nations System Standing Committee on Nutrition and the United Nations Children's Fund;World Health Organization: Geneva, Switzerland. 2007.

7. UNICEF, WHO, UNESCO, NUFPA, UNDP, UNAIDS, et al. Facts for Life, Fourth edition. 2010.

8. WHO. Essential nutrition actions: improving maternal, newborn, infant and young child health and nutrition. 2013.

9. Todd B, Bantirgu H, Solomon B, Demese C, Tefera B, Ayele G, et al. An assessment of the causes of malnutrition in Ethiopia, A contribution to the formulation of a National Nutrition Strategy for Ethiopia. 2005. 
437

438

439

440

441

442

443

444

445

446

447

448

449

450

451

452

453

454

455

456

457

458

459

460

461

462

463

464

465

466

467

468

469

470

471

472

473

474

475

476

477

478

479

480

481

10. Bhutta ZA, Das JK, Rizvi A, Gaffey MF, Walker N, Horton S, et al. Evidence-based interventions for improvement of maternal and child nutrition: what can be done and at what cost? The lancet. 2013;382(9890):452-77.

11. Collins S, Yates R. The Need to Update the Classification of Acute Malnutrition. [Letter] 2003;362((9379)).

12. Sphere-handbook. Sphere Handbook: Humanitarian Charter and Minimum Standards in Humanitarian Response: PRACTICAL ACTION; 2018.

13. WHO. Updates on the Management of Severe Acute Malnutrition in Infants and Children. World Health Organization: Geneva, Switzerland, . 2013.

14. FMOH. Protocol for the management of severe acute malnutrition, Federal Ministry of Health, Ethiopia. 2007.

15. Bhutta ZA, Ahmed T, Black RE, Cousens S, Dewey K, Giugliani E, et al. What works? Interventions for maternal and child undernutrition and survival. The lancet. 2008;371(9610):417-40.

16. Collins S. Treating severe acute malnutrition seriously. Arch Dis Child. 2007;92:453-61.

17. Tekeste A, Wondafrash M, Azene G, Deribe K. Cost effectiveness of community-based and inpatient therapeutic feeding programs to treat severe acute malnutrition in Ethiopia. http://www.resource-allocation.com/content/10/1/4. Cost Effectiveness and Resource Allocation. 2012;10(4).

18. Charulatha Banerjee, Hoq M, Matin E. Follow-up status of children with SAM treated with RUTF in peri-urban and rural Northern Bangladesh. 2016.

19. Chang CY, Trehan I, Wang RJ, Thakwalakwa C, Maleta K, Deitchler M, et al. Children Successfully Treated for Moderate Acute Malnutrition Remain at Risk for Malnutrition and Death in the Subsequent Year after Recovery. J Nutr 2013;143:215-20, .

20. Tadesse E, Worku A, Berhane Y, Ekström EC. An integrated community-based outpatient therapeutic feeding programme for severe acute malnutrition in rural Southern Ethiopia: Recovery, fatality, and nutritional status after discharge. Maternal \& child nutrition. 2018;14(2):e12519.

21. Concern-Worldwide. Evaluation of National Community management of acute malnutrition Programme, Ethiopia. 2010.

22. Stobaugh HC, Mayberry A, McGrath M, Bahwere P, Zagre NM, Manary MJ, et al. Relapse after severe acute malnutrition: A systematic literature review and secondary data analysis. Maternal \& child nutrition. 2019;15(2):e12702.

23. CSA. Federal Democratic Republic of Ethiopia, Central Statistical Agency, Population Projection of Ethiopia for All Regions At Wereda Level from 2014 - 2017. . 2013.

24. South-Gondar. South Gondar Zone Health Department annual report 2017/18.

25. Cogill B. Anthropometric indicators measurement guide. 2003.

26. Coates J, Swindale A, Bilinsky P. Food and Nutrition Technical Assistance Project (FANTA): Household Food Insecurity Access Scale (HFIAS) for Measurement of Food Access: Indicator Guide (v. 3). Washington, DC: Food and Nutrition Technical Assistance Project. 2007.

27. WHO. Child growth standard, Department of Nutrition for Health and Development. 2007.

28. Efrem T, Meskele L, Sahle S, Zerihun B, Daniel GD, Mohammed AY. Treatment outcome of children with severe acute malnutrition admitted to therapeutic feeding centers in Southern Region of Ethiopia Ethiop J Health Dev. 2010;24(3):234-8.

Peer) reviewing PDF | (2019:08:40536:2:0:NEW 8 Dec 2019) 
482

483

484

485

486

487

488

489

490

491

492

493

494

495

496

497

498

499

500

501

502

503

504

505

506

507

508

509

510

511

512

513

514

515

516

517

518

519

520

521

522

523

524

525

526

29. Indi Trehan, Somalee Banerjee, Ellen Murray, Kelsey N. Ryan, Chrissie Thakwalakwa, Kenneth M. Maleta, et al. Extending Supplementary Feeding for Children Under Five with Moderate Acute Malnutrition Leads to Lower Relapse rates. J Pediatr Gastroenterol Nutr. 2015;60(4):544-9.

30. Rozin P, Vollmecke TA. Food likes and dislikes. Annual review of nutrition. 1986;6(1):43356.

31. Alcock J, Maley CC, Aktipis CA. Is eating behavior manipulated by the gastrointestinal microbiota? Evolutionary pressures and potential mechanisms. Bioessays. 2014;36(10):9409.

32. Smith MI, Yatsunenko T, Manary MJ, Trehan I, Mkakosya R, Cheng J, et al. Gut microbiomes of Malawian twin pairs discordant for kwashiorkor. Science. 2013;339(6119):548-54.

33. Maust Amanda, Aminata S Koroma, Caroline Abla, Nneka Molokwu, Kelsey N Ryan, Lauren Singh, et al. Severe and Moderate AcuteMalnutrition Can Be Successfully Managed with an Integrated Protocol in Sierra Leone. The Journal of Nutrition. 2015:1-6.

34. Mgongo M, Chotta NA, Hashim TH, Uriyo JG, Damian DJ, Stray-Pedersen B, et al. Underweight, Stunting and Wasting among Children in Kilimanjaro Region, Tanzania; a Population-Based Cross-Sectional Study. International journal of environmental research and public health. 2017;14(5):509.

35. Udoh E, Amodu O. Complementary feeding practices among mothers and nutritional status of infants in Akpabuyo Area, Cross River State Nigeria. Nigeria: . SpringerPlus. 2016;5.

36. Poda GG, Hsu C-Y, Chao JC-J. Factors associated with malnutrition among children $<5$ years old in Burkina Faso: evidence from the Demographic and Health Surveys IV 2010. International Journal for Quality in Health Care. 2017;29(7):901-8.

37. Demissie S, Worku A. Magnitude and Factors Associated with Malnutrition in Children 6-59 Months of Age in Pastoral Community of Dollo Ado District, Somali Region, Ethiopia. Science Journal of Public Health. 2013;1(4):175-83.

38. Taye A, Wolde T, Seid A. Under-nutrition and related factors among children aged 6-59 months in Gida Ayana District, Oromiya region, West Ethiopia: a community based quantitative study. J Nutr Food Sci. 2016;6(5):1-12.

39. Kumi-Kyereme A, Amo-Adjei J. Household wealth, residential status and the incidence of diarrhoea among children under-five years in Ghana. Journal of Epidemiology and Global Health. 2016;6(3):131-40.

40. Chowdhury MRK, Rahman MS, Khan MMH, Mondal MNI, Rahman MM, Billah B. Risk factors for child malnutrition in Bangladesh: A multilevel analysis of a nationwide population-based survey. The Journal of pediatrics. 2016;172:194-201. e1.

41. Talukder B. Regional Differences of Child Under-Nutrition in Bangladesh. Finnish Yearbook of Population Research. 2013;48:189-201.

42. Nzala SH, Siziya S, Babaniyi O, Songolo P, Muula AS, Rudatsikira E. Demographic, cultural and environmental factors associated with frequency and severity of malnutrition among Zambian children less than five years of age. Journal of Public Health and Epidemiology. 2011;3(8):362-70.

43. Gone T, Lemango F, Eliso E, Yohannes S, Yohannes T. The association between malaria and malnutrition among under-five children in Shashogo District, Southern Ethiopia: a casecontrol study. Infectious diseases of poverty. 2017;6(1):9. 
527

528

529

530

531

532

533

534

535

536

537

538

539

540

541

542

543

544

545

546

547

548

549

550

551

552

553

554

555

556

557

558

559

560

561

562

563

564

565

566

567

568

569

570

571

44. Seid A, Seyoum B, Mesfin F. Determinants of Acute Malnutrition among Children Aged 659 Months in Public Health Facilities of Pastoralist Community, Afar Region, Northeast Ethiopia: A Case Control Study. Journal of nutrition and metabolism. 2017;2017.

45. Mishra K, Kumar P, Basu S, Rai K, Aneja S. Risk factors for severe acute malnutrition in children below 5 y of age in India: a case-control study. The Indian Journal of Pediatrics. 2014;81(8):762-5.

46. Bantamen G, Belaynew W, Dube J. Assessment of factors associated with malnutrition among under five years age children at Machakel Woreda, Northwest Ethiopia: a case control study. Journal of Nutrition \& Food Sciences. 2014;4(1):1.

47. Guldan GS, Fan H-C, Ma X, Ni Z-Z, Xiang X, Tang M-Z. Culturally appropriate nutrition education improves infant feeding and growth in rural Sichuan, China. The Journal of nutrition. 2000;130(5):1204-11.

48. Daba A, Ersado M. Impact of Community Based Management of Acute Malnutrition Integrated Nutrition Education on Infant and Young Child Feeding Knowledge and Practice of Mothers or Caregivers in Dilla Zuria Woreda, Southern Ethiopia: A Quasi Experimental Study. Fam Med Med Sci Res. 2015;4(190):2.

49. Appoh LY, Krekling S. Maternal nutritional knowledge and child nutritional status in the Volta region of Ghana. Maternal \& child nutrition. 2005;1(2):100-10.

50. Egata G, Berhane Y, Worku A. Predictors of acute undernutrition among children aged 6 to 36 months in east rural Ethiopia: a community based nested case - control study. BMC Pediatrics. 2014;14(91).

51. Awoke A, Ayana M, Gualu T. Determinants of severe acute malnutrition among under five children in rural Enebsie Sarmidr District, East Gojjam Zone, North West Ethiopia, 2016. BMC Nutrition. 2018;4(1):4.

52. Ambadekar N, Zodpey S. Risk factors for severe acute malnutrition in under-five children: a case-control study in a rural part of India. Public health. 2017;142:136-43.

53. Tewabe T, Mandesh A, Gualu T, Alem G, Mekuria G, Zeleke H. Exclusive breastfeeding practice and associated factors among mothers in Motta town, East Gojjam zone, Amhara Regional State, Ethiopia, 2015: a cross-sectional study. International breastfeeding journal. 2016;12(1):12.

54. Qadri HA, Srivastav SK. Under-nutrition more in male children: a new study. International Journal of Research in Medical Sciences. 2017;3(11):3363-6.

55. Sanghvi J, Mehta S, Kumar R. Predicators for Weight Gain in Children Treated for Severe Acute Malnutrition: A Prospective Study at Nutritional Rehabilitation Center. . Hindawi Publishing Corporationhttp. 2014:1-5.

56. Bisrat G, Kulkarni U. Assessment of the Nutritional Status and Associated Factors of Orphans and Vulnerable Preschool Children on Care and Support from Nongovernmental Organizations in Hawassa Town, Southern Ethiopia. Global Journal of Medical Research. 2017.

57. Huang Z, Liu Y, Qi G, Brand D, Zheng S. Role of Vitamin A in the Immune System. Journal of clinical medicine. 2018;7(9):258.

58. WHO. Guideline: Vitamin A supplementation in infants and children 6-59 months of age. Geneva, World Health Organization. 2011.

59. WHO, UNICEF. Global strategy for infant and young child feeding. 2003 ISBN 924156221 8.

Peer) reviewing PDF | (2019:08:40536:2:0:NEW 8 Dec 2019) 
572

573

574

575

576

577

578

579

580

581

582

583

584

585

586

587

588

589

590

591

592

593

594

595

596

597

598

599
60. Nzala SH, Siziya S, Babaniyi O, Songolo P, Muula AS, Rudatsikira E. Demographic, cultural and environmental factors associated with frequency and severity of malnutrition among Zambian children less than five years of age. J Publ Health Epidemiol. 2011;3(8):362-70.

61. Cronin A, Sebayang S, Torlesse H, Nandy R. Association of safe disposal of child feces and reported diarrhea in Indonesia: need for stronger focus on a neglected risk. International journal of environmental research and public health. 2016;13(3):310.

62. Burza S, R M, Marino E, Sunyoto T, C S, M T, et al. Seasonal effect and long-term nutritional status following exit from a Community-Based Management of Severe Acute Malnutrition program in Bihar, India. European Journal of Clinical Nutrition. 2016;70:43744.

63. Ajao K, Ojofeitimi E, Adebayo A, Fatusi A, Afolabi O. Influence of Family Size, Household Food Security Status, and Child Care Practices on the Nutritional Status of Under-five Children in Ile-Ife, Nigeria Afr J Reprod Health. 2010;14(4):123 - 32.

64. Miskir A, Godana W, Girma M, G/Miskel F. Determinants of Acute Malnutrition among Under-Five Children in Karat Town Public Health Facilities, Southern Ethiopia: A Case Control Study. Quality in Primary Care 2017;25(4):242-52.

65. Dodos J, Altare C, Bechir M, Myatt M, Pedro B, Bellet F, et al. Individual and household risk factors of severe acute malnutrition among under-five children in Mao, Chad: a matched case-control study. Archives of Public Health. 2018;76(1):35.

66. Frozanfar MK, Yoshida Y, Yamamoto E, Reyer JA, Dalil S, Rahimzad AD, et al. Acute malnutrition among under-five children in Faryab, Afghanistan: prevalence and causes. Nagoya journal of medical science. 2016;78(1):41.

67. WHO, UNICEF, USAID. Improving nutrition outcomes with better water, sanitation and hygiene: practical solutions for policies and programmes. 2015.

68. Brown J, Cairncross S, Ensink JH. Water, sanitation, hygiene and enteric infections in children. Archives of disease in childhood. 2013;98(8):629-34. 
Table $\mathbf{1}$ (on next page)

Demographic and maternity related 
1 Table 1: Socioeconomic/demographic and maternity related characteristics of mothers of 2 children age 6-59 month in South Gondar Zone, Amhara Region, Ethiopia 2017/18

Variable

Respondent age in yrs.
Variable

\section{Recovered, $\mathbf{n}$}

$(\%)$
Comparison, $n \quad \mathbf{X}^{2}$

$(\%)$

$19(2.7)$

$323(45.7)$

$294(41.6)$

$67(9.5)$

$4(0.6)$

District

Child age in month

\begin{tabular}{l}
\hline $20-29$ \\
\hline $30-39$ \\
\hline $40-49$ \\
\hline $50+$
\end{tabular}

Ebnat

$301(42.6)$

Tack Gayint

Lay Gayint

308 (43.6) $27(3.8)$

6-11

98 (13.9)

$50(7.1)$

$$
\text { 12-23 }
$$

24-35

$36-47$

48-59

$314(44.4)$

$234(33.1)$

$73(10.3)$

$36(5.1)$

\begin{tabular}{|c|c|c|c|c|}
\hline Sex of child (female) & $346(48.9)$ & $324(45.8)$ & 1.373 & 0.241 \\
\hline HH headship (Male) & $662(93.6)$ & $618(87.4)$ & 15.90 & $<0.001$ \\
\hline Respondent education status (No formal) & $580(82.0)$ & $556(78.6)$ & 2.579 & 0.108 \\
\hline Respondent religion (Orthodox) & $657(92.9)$ & $654(92.5)$ & 0.094 & 0.759 \\
\hline Marital status (Currently married) & $661(93.5)$ & $633(89.5)$ & 7.139 & 0.008 \\
\hline HH family size $(>=5)$ & $400(56.6)$ & $373(52.8)$ & 2.08 & 0.149 \\
\hline Total under 5 children $(2+)$ & $166(23.5)$ & $153(21.6)$ & 0.684 & 0.408 \\
\hline Respondent occupation (Farming) & $573(81.0)$ & $542(76.7)$ & 4.076 & 0.043 \\
\hline HH currently on Food Aid (Yes) & $189(26.7)$ & $217(30.7)$ & 2.709 & 0.100 \\
\hline Own at least one $\mathrm{HH}$ effect* (Yes) & $135(19.1)$ & $183(25.9)$ & 9.347 & 0.002 \\
\hline HH own agricultural land (Yes) & $655(93.3)$ & $596(88.8)$ & 26.78 & $<0.001$ \\
\hline HH own farm animals (Yes) & $654(92.5)$ & $590(83.5)$ & 27.39 & $<0.001$ \\
\hline HH food insecure (Yes) & $164(23.2)$ & $178(25.2)$ & 0.756 & 0.358 \\
\hline Decision maker on $\mathrm{HH}$ expenditure (together) & $300(42.4)$ & $241(34.1)$ & 10.44 & 0.001 \\
\hline Attend ANC during this pregnancy (Yes) & $564(79.8)$ & $595(84.2)$ & 4.598 & 0.032 \\
\hline Place of delivery (Home) & $244(34.5)$ & $236(33.4)$ & 0.202 & 0.653 \\
\hline $\begin{array}{l}\text { Additional food consumption at } \\
\text { pregnancy/lactation (Yes) }\end{array}$ & $432(61.1)$ & $407(57.6)$ & 1.832 & 0.176 \\
\hline Currently on Family Planning (Yes) & $564(79.8)$ & $468(66.2)$ & 33.06 & $<0.001$ \\
\hline Birth interval in month ( $<24$ month) & $104(14.8)$ & $82(11.6)$ & 2.996 & 0.083 \\
\hline Birth order $(3+)$ & $412(58.3)$ & $387(54.7)$ & 1.789 & 0.180 \\
\hline Home to HP walk in minute $(<=30)$ & $305(43.1)$ & $383(54.2)$ & 17.22 & $<0.001$ \\
\hline
\end{tabular}




\begin{tabular}{lcccc}
\hline Wealth Quintile (Lowest) & $105(15.0)$ & $183(26.1)$ & 29.21 & $<0.001$ \\
\hline Key: HH -household, ANC-Antenatal care, HP-health post, & $*$ (radio, & TV, mobile, refrigerator) &
\end{tabular}
3 
Table 2 (on next page)

Child feeding/caring and housing conditions 
1 Table 2: Child feeding/caring, housing conditions and related characteristics of mothers of children age 6-59 mothers in South Gondar Zone, Amhara Region, Ethiopia 2017/18

\begin{tabular}{|c|c|c|c|c|}
\hline Variables & $\begin{array}{c}\text { Recovere } \\
\text { d, } \\
\text { n (\%) }\end{array}$ & $\begin{array}{c}\text { Compariso } \\
\text { n, } \\
\text { n }(\%)\end{array}$ & $\mathbf{X}^{2}$ & $\begin{array}{c}\text { p- } \\
\text { value }\end{array}$ \\
\hline $\begin{array}{l}\text { BF initiation immediately within an hour after } \\
\text { birth }\end{array}$ & $447(63.2)$ & $461(65.5)$ & 0.784 & 0.376 \\
\hline Colostrum provided (Yes) & $516(73.0)$ & $501(71.2)$ & 0.580 & 0.446 \\
\hline Prelactal feeding (Yes) & $47(6.6)$ & $35(5.0)$ & 1.864 & 0.172 \\
\hline Child currently on breastfeeding (Yes) & $559(79.1)$ & $572(80.9)$ & 0.747 & 0.388 \\
\hline $\begin{array}{l}\text { Complementary feeding as } \text { WHO recommended(2- } \\
\text { 4times/day) }\end{array}$ & $614(86.8)$ & $635(89.8)$ & 3.026 & 0.082 \\
\hline $\begin{array}{l}\text { Prepare food to children other than family diet } \\
\text { (Yes) }\end{array}$ & $431(61.0)$ & $391(55.3)$ & 4.649 & 0.031 \\
\hline Trained in child food preparation (Yes) & $406(57.4)$ & $372(52.6)$ & 3.303 & 0.068 \\
\hline Child vaccinated for measles (Yes) & $678(95.9)$ & $602(85.1)$ & 47.62 & 0.0001 \\
\hline Vitamin A received in the last 6 months (Yes) & $536(75.8)$ & $438(62.0)$ & 31.69 & 0.0001 \\
\hline Deworming given in the past 6 months (Yes) & $150(21.2)$ & $140(19.8)$ & 0.434 & 0.510 \\
\hline History of diarrhea last 2 wks. (Yes) & $61(8.6)$ & $75(10.6)$ & 1.595 & 0.207 \\
\hline HH water source type (Improved) & $397(56.2)$ & $408(57.7)$ & 0.349 & 0.555 \\
\hline $\mathrm{HH}$ latrine type (traditional pit latrine) & $625(88.4)$ & $609(86.1)$ & 1.630 & 0.202 \\
\hline HH dry waste disposed (open fields) & $360(50.9)$ & $345(48.8)$ & 0.636 & 0.425 \\
\hline HH child feces dispose practice (safe) & $388(54.9)$ & $424(60)$ & 3.494 & 0.053 \\
\hline Hand washing practice (good) & $400(56.6)$ & $388(54.9)$ & 0.143 & 0.521 \\
\hline Often wash hands with (water only) & $352(49.8)$ & $364(51.5)$ & 0.777 & 0.678 \\
\hline Time to water source ( $<30$ minute walk) & $280(17.3)$ & $309(43.7)$ & 2.447 & 0.118 \\
\hline Other family member on SAM treatment (yes) & $19(2.7)$ & $6(0.8)$ & 6.882 & 0.009 \\
\hline \multicolumn{5}{|l|}{ Average OTP treatment stay in weeks $(8 . .0 \pm 1.7)$} \\
\hline $\begin{array}{l}\text { Average duration after recovery in month } \\
(4.8 \pm 1.9)\end{array}$ & & NA & & \\
\hline Duration since recovery in month $(<=3)$ & $234(33.1)$ & NA & & \\
\hline
\end{tabular}


Table 3 (on next page)

Prevalence of acute malnutrition 
1 Table 3: Nutritional status of children age 6-59 months following exit from CMAM and the

\begin{tabular}{|c|c|c|c|c|c|c|}
\hline \multirow[t]{2}{*}{ Indicators } & & \multicolumn{2}{|c|}{ Recovered } & \multicolumn{2}{|c|}{ Comparison group } & \multirow[b]{2}{*}{$P$ value } \\
\hline & & $\mathrm{n}(\%)$ & $95 \% \mathrm{CI}$ & $\mathrm{n}(\%)$ & $95 \% \mathrm{CI}$ & \\
\hline \multirow[t]{2}{*}{ MUAC $(\mathrm{cm})$} & Normal $(\geq 12.5)$ & $464(65.6)$ & $62.0-69.1$ & $518(73.3)$ & $69.8-76.5$ & \multirow[b]{2}{*}{0.002} \\
\hline & Wasted $(<12.5)$ & $243(34.4)$ & $30.9-38.0$ & $189(26.7)$ & $23.5-30.2$ & \\
\hline \multirow[t]{2}{*}{ WHZ } & Normal $(\geq-2 S D)$ & $484(68.5)$ & 64.9-71.9 & $542(76.7)$ & 73.4-79.7 & \multirow[b]{2}{*}{$<0.001$} \\
\hline & Wasted <-2SD) & $223(31.5)$ & $28.1-35.1$ & $165(23.3)$ & $20.3-26.6$ & \\
\hline \multirow[t]{2}{*}{ HAZ } & Normal ( $\geq-2 S D)$ & $234(33.1)$ & $29.6-36.7$ & $295(41.7)$ & $38.1-45.5$ & \multirow[b]{2}{*}{0.001} \\
\hline & Stunted $(<-2 \mathrm{SD})$ & $473(66.9)$ & $63.3-70.4$ & $412(58.3)$ & $54.5-61.9$ & \\
\hline \multirow[t]{3}{*}{ WAZ } & Normal $(\geq-2 S D)$ & $345(48.8)$ & $45.1-52.6$ & $430(60.8)$ & $57.1-64.4$ & \multirow{3}{*}{$<0.001$} \\
\hline & Underweight <- & $362(51.2)$ & $47.4-$ & $277(39.2)$ & 35.6 & \\
\hline & 2SD) & & 54.9 & & 42.9 & \\
\hline
\end{tabular}

Key: MUAC (mid upper arm circumference), WHZ (Weight for Height Z score), HAZ (Height for age Z score), WAZ (Weight for Age Z score)

3

4

5

6 


\section{Table 4(on next page)}

Factors associated with acute malnutrition 
Table 4: Factors associated with acute malnutrition of children age 6-59 mothers in South Gondar Zone, Amhara region, Ethiopia, 2017/18

\begin{tabular}{|c|c|c|c|c|c|c|c|c|c|}
\hline \multirow[t]{2}{*}{ Variables } & & \multicolumn{4}{|c|}{$\begin{array}{l}\text { Acute malnutrition in discharge as recovered } \\
\text { children }\end{array}$} & \multicolumn{4}{|c|}{ Acute malnutrition in comparison group } \\
\hline & & Yes & No & COR $(95 \% \mathrm{CI})$ & AOR $(95 \% \mathrm{CI})$ & Yes & No & COR $(95 \% \mathrm{CI})$ & AOR $(95 \% \mathrm{CI})$ \\
\hline \multirow[t]{3}{*}{ District } & Ebnat & 125 & 176 & $4.26(2.31-7.85)$ & $3.67(1.86-7.24)$ & 80 & 262 & $1.69(0.92-3.09)$ & $1.65(0.86-3.14)$ \\
\hline & $\begin{array}{l}\text { Tach- } \\
\text { Gayint }\end{array}$ & 104 & 204 & $3.06(1.66-5.65)$ & $2.36(1.20-4.69)$ & 94 & 173 & $3.01(1.64-5.50)$ & $2.49(1.29-4.81)$ \\
\hline & $\begin{array}{l}\text { Lay } \\
\text { Gayint }\end{array}$ & 14 & 84 & 1 & 1 & 15 & 83 & 1 & 1 \\
\hline Sex & Female & 110 & 236 & 1 & 1 & 76 & 248 & 1 & 1 \\
\hline \multirow[t]{2}{*}{ Prelacteal feeding } & No & 215 & 445 & 1 & 1 & & & & \\
\hline & Yes & 28 & 19 & $3.05(1.67-5.59)$ & $2.56(1.28-5.12)$ & & & & \\
\hline $\begin{array}{l}\text { Vit A supplement } \\
\text { in past } 6 \text { months }\end{array}$ & No/don't & 91 & 80 & $2.87(2.02-4.10)$ & $2.13(1.42-3.19)$ & & & & \\
\hline $\begin{array}{l}\text { Additional food at } \\
\text { pregnancy/lactation }\end{array}$ & Yes & 119 & 313 & 1 & 1 & & & & \\
\hline \multirow[t]{2}{*}{ Colostrum feeding } & No & 82 & 109 & $1.66(1.18-2.34)$ & $1.51(1.01-2.26)$ & 74 & 129 & $1.97(1.38-2.81)$ & $1.66(1.23-2.45)$ \\
\hline & Yes & 161 & 355 & 1 & 1 & 113 & 388 & 1 & 1 \\
\hline \multirow{2}{*}{$\begin{array}{l}\text { Respondent age in } \\
\text { yrs. }\end{array}$} & $<=30$ & 97 & 245 & 1 & 1 & & & & \\
\hline & $>30$ & 146 & 219 & $1.68(1.23-2.21)$ & $1.63(1.15-2.31)$ & & & & \\
\hline \multirow[t]{2}{*}{ HH food security } & No & & & & & 68 & 110 & $2.08(1.45-3.00)$ & $1.59(1.06-2.38)$ \\
\hline & Yes & & & & & 121 & 408 & 1 & 1 \\
\hline \multirow{2}{*}{$\begin{array}{l}\text { Good hand washing } \\
\text { practice }\end{array}$} & No & & & & & 100 & 219 & $1.53(1.10-2.14)$ & $1.55(1.08-2.21)$ \\
\hline & Yes & & & & & 89 & 299 & 1 & 1 \\
\hline
\end{tabular}




\begin{tabular}{lc|cccc}
\hline Birth interval in & $<24$ & 31 & 51 & $1.80(1.11-2.91)$ & $1.92(1.15-3.19)$ \\
\cline { 2 - 6 } month & $>=24$ & 158 & 467 & 1 & 1 \\
\hline Home to HP by foot & $<=30$ & 83 & 300 & 1 & 1 \\
\cline { 2 - 7 } walk in minute & $>30$ & 106 & 218 & $1.76(1.26-2.46)$ & $1.66(1.16-2.37)$ \\
\hline
\end{tabular}


Figure 1

Acute malnutrition in the two groups 


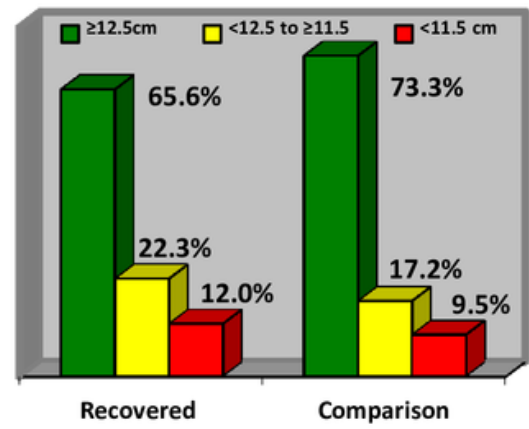

